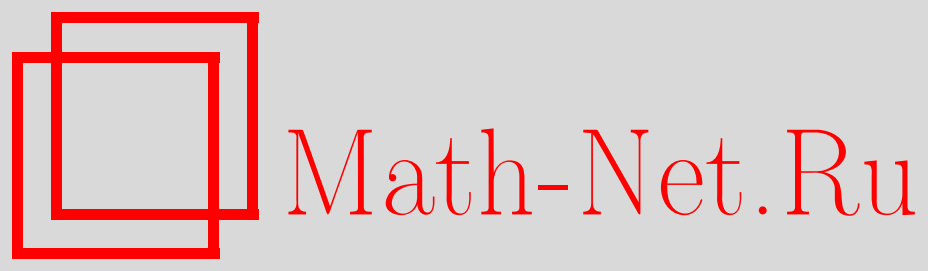

С. В. Керов, Анизотропные диаграммы Юнга и симметрические функции Джека, Функи. анализ и его прил., 2000, том 34, выпуск 1, 51-64

DOI: https://doi.org/10.4213/faa282

Использование Общероссийского математического портала MathNet.Ru подразумевает, что вы прочитали и согласны с пользовательским соглашением

http://www . mathnet.ru/rus/agreement

Параметры загрузки:

IP : 3.95 .254 .165

26 апреля 2023 г., 16:33:04

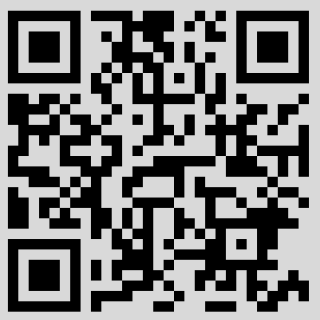


Функциональньй анализ и его приложения

2000, т. 34, вып. 1, с. 51-64

УДК 519.217, 517.986

\title{
Анизотропные диаграммы Юнга и симметрические функции Джека*
}

\author{
(C) 2000. C. В. Керов
}

Введение

Основное наблюдение, лежащее в основе этой статьи, состоит в том, что аналитическое понятие перемежаюшихся последовательностей является естественным обобшением комбинаторного понятия диаграммы Юнга (см. подробное изложение в [5] и приложения комбинаторных методов к асимптотическому анализу перемежающихся последовательностей в $[3,4])$. Мы покажем, что из простых аналитических фактов о перемежающихся последовательностях следует формула крюков для размерностей диаграмм Юнга (точнее, для отвечающих им представлений), а также формула для переходных вероятностей меры Планшереля бесконечной симметрической группы. Более общим образом, мы получаем переходные вероятности для семейства $z$-мер, введенных в [6] и составляющих деформацию меры Планшереля. Оба результата допускают непосредственное обобщение, в котором формула Пиери для функций Шура заменяется на аналогичную формулу для симметрических функций Джека.

Аналитический подход к формуле крюков для обычных размерностей был предметом работ $[1,8,11]$. Идея этой статьи состоит в том, что соображения из [1] доставляют без всяких дополнительных усилий аналогичные факты для размерностей, связанных с симметрическими функциями Джека. Единственное отличие состоит в том, что мы отождествляем диаграммы Юнга с перемежающимися последовательностями иначе, принимая во внимание параметр $\alpha$ полиномов Джека. Грубо говоря, обычные диаграммы Юнга следует растянуть в $\alpha$ раз вдоль горизонтальной оси. Можно также сказать, что новые «диаграммы» построены из прямоугольных «клеток» ширины $\alpha$ и единичной высоты вместо единичных квадратных клеток для обычных диаграмм Юнга. Мы называем их анизотропньми диаграммами Юнга.

Работа построена следующим образом. В начале первого параграфа мы напоминаем, как диаграммы Юнга отождествляются с парами перемежающихся последовательностей. В $\S 2$ мы используем простейшие дроби для того чтобы связать с парой перемежающихся последовательностей два дискретных вероятностных распределения. Как объясняется в $\S \S 3-4$, эти распределения обобщают переходное и копереходное распределения

$$
p_{\lambda}(\Lambda)=\frac{\operatorname{dim} \Lambda}{|\Lambda| \operatorname{dim} \lambda}, \quad q_{\Lambda}(\lambda)=\frac{\operatorname{dim} \lambda}{\operatorname{dim} \Lambda}, \quad \lambda \nearrow \Lambda,
$$

*Работа выполнена при поддержке ФЦП «Интеграция» (грант 326.53) и Института математических исследований (Беркли). 
определенные в комбинаторных терминах. В 55 мы находим моменты переходного распределения, связанного с парой перемежающихся последовательностей, и показываем, что первые три момента зависят не от деталей диаграммы, а только от числа ее клеток. В $\S 6$ дано краткое аналитическое доказательство $\alpha$-формулы крюков, впервые найденной Стенли в [10]. В заключительном $\S 7$ мы устанавливаем наш второй основной результат, теорему 7.2. Это обобщение на случай симметрических полиномов Джека семейства центральных мер на решетке Юнга (или, что эквивалентно, семейства сферических функций на бесконечной симметрической группе), введенного в [6]. Подробности о центральных мерах на графе Джека см. в [13].

Благодарности. Я весьма ценю многочисленные обсуждения с Г. И. Ольшанским взаимоотношений между комбинаторикой и анализом диаграмм Юнга вообще и комбинаторики полиномов Джека в частности. Я также благодарен А. М. Вершику, А. Ю. Окунькову и С. В. Фомину за их интерес к этой работе. Статья была завершена во время визита в MSRI, Беркли. Мой приятный долг - поблагодарить организаторов комбинаторной программы 1997 г. за приглашение и финансовую поддержку.

\section{§1. Перемежающиеся последовательности и диаграммы Юнга}

Цель этого параграфа - объяснить, что диаграммы Юнга удобно рассматривать как целочисленные перемежающиеся последовательности. Другие аргументы в пользу этого тезиса см. в [3-5].

Мы всегда будем перечислять элементы заданного конечного множества $\left\{x_{1}, \ldots, x_{d}\right\}$ различных вещественных чисел в возрастающем порядке и отождествлять это множество с соответствующей последовательностью.

ОПРЕДЕлЕНИЕ 1.1. Два множества (или две возрастающие последовательности) $y_{1}, \ldots, y_{d-1}$ и $x_{1}, \ldots, x_{d-1}, x_{d}$ называются перемежающимися, если

$$
x_{1}<y_{1}<x_{2}<\cdots<x_{d-1}<y_{d-1}<x_{d} .
$$

Число $c=\sum x_{k}-\sum y_{k}$ называется иентром этих перемежающихся последовательностей.

С каждой парой перемежающихся последовательностей (1.1) мы связываем кусочно линейную непрерывную функцию $v=\omega(u)$, такую, что

(i) $\omega^{\prime}(u)=+1$, если $x_{k}<u<y_{k}, k=1, \ldots, d-1$;

(ii) $\omega^{\prime}(u)=-1$, если $y_{k}<u<x_{k+1}, k=1, \ldots, d-1$;

(iii) $\omega(u)=|u-c|$, если $u<x_{1}$ или $u>x_{d}$.

Можно легко убедиться, что такая функция существует и однозначно определяется условиями (i)-(iii). Действительно, из (iii) следует, что $\omega\left(x_{1}\right)=c-x_{1}$ и $\omega\left(x_{d}\right)=x_{d}-c$. Согласно (i) и (ii), мы знаем, что $\omega\left(y_{k}\right)-\omega\left(x_{k}\right)=y_{k}-x_{k}$ и $\omega\left(x_{k+1}\right)-\omega\left(y_{k}\right)=y_{k}-x_{k+1}$. Отсюда вытекает, что

$$
\begin{aligned}
& \omega\left(x_{k}\right)=\sum_{i=1}^{k-1}\left(y_{i}-x_{i}\right)+\sum_{j=k}^{d-1}\left(x_{j+1}-y_{j}\right), \\
& \omega\left(y_{k}\right)=\sum_{i=1}^{k}\left(y_{i}-x_{i}\right)+\sum_{j=k}^{d-1}\left(x_{j+1}-y_{j}\right) .
\end{aligned}
$$



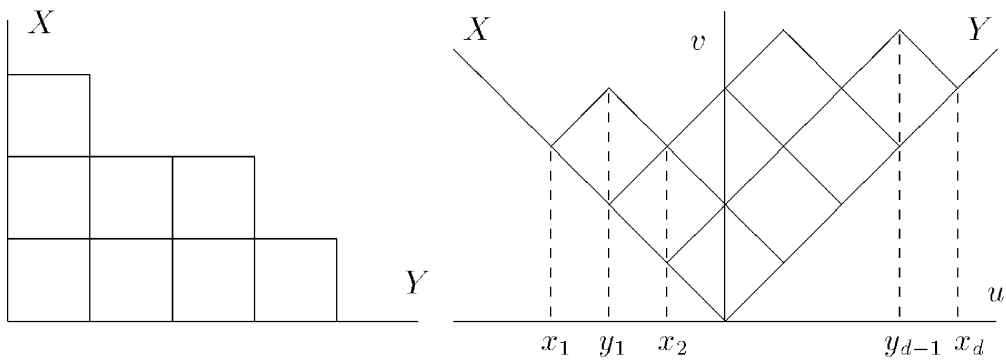

Рис. 1. Диаграмма Юнга как пара перемежающихся последовательностей

Область

$$
S_{\omega}=\left\{(u, v) \in \mathbb{R}^{2}:|u-c| \leqslant v<\omega(u)\right\}
$$

между графиками функций $v=\omega(u)$ и $v=|u-c|$ напоминает форму диаграммы Юнга (см. рис. 1). Мы будем говорить, что $\omega$ - это диаграмма перемежающихся последовательностей (1.1), а область (1.3) — ее форма. Площадь диаграммы определяется формулой

$$
A(\omega)=\sum_{i<j}\left(y_{i}-x_{i}\right)\left(x_{j}-y_{j-1}\right) .
$$

Обозначим через $\mathbb{I}_{d}$ пространство перемежающихся последовательностей (1.1) с $2 d-1$ элементами и через $\mathbb{I}=\bigcup \mathbb{I}_{d}$ множество всех перемежающихся последовательностей. Топология пространства II задается равномерной сходимостью соответствуюших диаграмм.

Форма истинной диаграммы Юнга $\lambda=\left(\lambda_{1}, \ldots, \lambda_{m}\right)$ однозначно определяется содержаниями $y_{1}, \ldots, y_{d-1}$ ее угловых клеток и содержаниями $x_{1}, \ldots, x_{d-1}, x_{d}$ угловых клеток дополнения диаграммы $\lambda$ в $\mathbb{R}_{+}^{2}$. (Напомним, что содержание клетки $b=(i, j)$ на пересечении $i$-й строки и $j$-го столбца определяется как $c(b)=j-i$. Удобно считать, что любая точка плоскости с координатами $(u, v)$ имеет содержание $c(u, v)=v-u$. Тогда содержание клетки совпадает с содержанием ее середины.) Последовательности целых чисел $y_{1}, \ldots, y_{d-1}$ и $x_{1}, \ldots$, $x_{d-1}, x_{d}$ перемежаются. Центр такой пары всегда нулевой, $c=\sum x_{k}-\sum y_{k}=0$.

Обратно, каждая пара перемежающихся последовательностей (1.1) с целыми элементами и нулевым центром представляет истинную диаграмму Юнга. Число строк $m$ в такой диаграмме равно $-x_{1}$, а $\lambda_{1}=x_{d}$ - длина первой строки. Число клеток $|\lambda|=A(\omega)$ равно площади ассоциированной диаграммы $\omega$.

Итак, множество $\mathbb{Y}$ диаграмм Юнга можно рассматривать как решетку целых точек в пространстве $\mathbb{I}_{0}$ перемежающихся последовательностей с нулевым центром.

Обычно диаграмму Юнга $\lambda$ рисуют, располагая строки горизонтально, в порядке убывания длин, так что график соответствующей кусочно-линейной функции следует повернуть на $45^{\circ}$. Содержания клеток горизонтальной части границы диаграммы $\lambda$ заполняют интервалы $\left(x_{i}, y_{i}\right)$, а координаты вертикальных частей границы - интервалы $\left(y_{i}, x_{i+1}\right), i=1, \ldots, d-1$. 


\section{§2. Переходные и копереходные распределения перемежающихся последовательностей}

В этом параграфе мы связываем с каждой парой перемежающихся последовательностей два дискретных вероятностных распределения. В частном случае последовательностей, соответствующих диаграмме Юнга, эти распределения совпадают с переходным и копереходным распределениями для меры Планшереля бесконечной симметрической группы. Все факты, которые мы здесь напоминаем, хорошо известны.

Для заданной пары (1.1) множеств $X=\left(x_{1}, \ldots, x_{d-1}, x_{d}\right)$ и $Y=\left(y_{1}, \ldots, y_{d-1}\right)$ мы обозначаем через $P(u)=\prod\left(u-x_{k}\right)$ и $Q(u)=\prod\left(u-y_{k}\right)$ приведенные многочлены с соответствующими корнями. Разложим рациональную дробь $R(u)=$ $Q(u) / P(u)$ в сумму простейших дробей,

$$
\frac{\left(u-y_{1}\right) \cdots\left(u-y_{d-1}\right)}{\left(u-x_{1}\right) \cdots\left(u-x_{d-1}\right)\left(u-x_{d}\right)}=\sum_{k=1}^{d} \frac{\mu_{k}}{u-x_{k}} .
$$

Умножая обе части на $u$ и беря предел при $u \rightarrow \infty$, мы получаем, что

$$
\sum_{k=1}^{d} \mu_{k}=1
$$

Лемма 2.1. Следующие два свойства дроби (2.1) эквивалентны:

(a) $x_{1}<y_{1}<x_{2}<\cdots<x_{d-1}<y_{d-1}<x_{d}$;

(b) $\mu_{1}>0, \ldots, \mu_{d}>0$.

ДокАЗАТЕльСТво. По формуле вычетов

$$
\mu_{k}=\frac{Q\left(x_{k}\right)}{P^{\prime}\left(x_{k}\right)}=\prod_{i<k} \frac{x_{k}-y_{i}}{x_{k}-x_{i}} \prod_{j>k} \frac{x_{k}-y_{j-1}}{x_{k}-x_{j}} .
$$

Ясно, что если корни и полюсы дроби $R(u)$ перемежаются, то все коэффициенты $\mu_{k}$ положительны.

Предположим теперь, что $\mu_{k}>0$ для всех $k=1, \ldots, d$. Если бы нашлись две соседние $x$-точки, не разделенные $y$-точкой, то соответствующие коэффициенты $\mu_{k}$ имели бы противоположные знаки. Следовательно, имеется ровно одна $y$-точка между каждыми соседними $x$-точками и последовательности перемежаются.

ОпРЕдЕлениЕ 2.2. Каждой паре перемежающихся последовательностей $x_{1}<$ $y_{1}<\cdots<y_{d-1}<x_{d}$ соответствует дискретное вероятностное распределение $\mu$ с весами

$$
\mu_{k}=\prod_{i=1}^{k-1} \frac{x_{k}-y_{i}}{x_{k}-x_{i}} \prod_{j=k+1}^{d} \frac{x_{k}-y_{j-1}}{x_{k}-x_{j}}
$$

в точках $x_{k}, k=1, \ldots, d$. Мы называем $\mu$ переходным распределением этой пары, а веса $\mu_{k}$ - ее переходными вероятностями. 
Другое распределение, связанное с парой перемежающихся последовательностей, возникает из разложения

$$
\frac{\left(u-x_{1}\right) \cdots\left(u-x_{d-1}\right)\left(u-x_{d}\right)}{\left(u-y_{1}\right) \cdots\left(u-y_{d-1}\right)}=u-c-\sum_{k=1}^{d-1} \frac{\nu_{k}}{u-y_{k}} .
$$

ЛЕмма 2.3. Коэффициент $c=c(\omega)$ в (2.3) совпадает с иентром диаграммь $\omega$, а сумма коэффициентов $\nu_{k}$ равна ее площади, $\sum \nu_{k}=A(\omega)$. Следуюшие два условия эквивалентни:

(a) $x_{1}<y_{1}<x_{2}<\cdots<x_{d-1}<y_{d-1}<x_{d}$;

(b) $\nu_{1}>0, \ldots, \nu_{d-1}>0$.

ДокАЗАТЕльство. Чтобы проверить первые два утверждения леммы, мы умножаем обе части равенства $(2.3)$ на многочлен $P(u)$ и сравниваем несколько первых коэффициентов. Пусть

$$
e_{k}(X)=\sum_{i_{1}<\cdots<i_{k}} x_{i_{1}} \cdots x_{i_{k}} \quad \text { и } \quad e_{k}(Y)=\sum_{i_{1}<\cdots<i_{k}} y_{i_{1}} \cdots y_{i_{k}}
$$

обозначают $k$-е элементарные симметрические функции от переменных $X=$ $\left(x_{1}, \ldots, x_{d}\right)$ и $Y=\left(y_{1}, \ldots, y_{d-1}\right)$ соответственно. С точностью до членов степени $d-3$,

$$
\begin{aligned}
& u^{d}-e_{1}(X) u^{d-1}+e_{2}(X) u^{d-2}-\cdots \\
& \quad=-u^{d-2} \sum \nu_{k}+\cdots+(u-c)\left(u^{d-1}-e_{1}(Y) u^{d-2}+e_{2}(Y) u^{d-3}-\cdots\right),
\end{aligned}
$$

так что $c=e_{1}(X)-e_{1}(Y)$ и $\sum \nu_{k}=-e_{2}(X)+e_{1}(X) e_{1}(Y)-e_{1}^{2}(Y)+e_{2}(Y)=A(\omega)$.

По формуле вычетов

$$
\nu_{k}=-\frac{P\left(y_{k}\right)}{Q^{\prime}\left(y_{k}\right)}=-\prod_{i}\left(y_{k}-x_{i}\right) \prod_{j \neq k}\left(y_{k}-y_{j}\right)^{-1} .
$$

Если последовательности $X$ и $Y$ перемежаются, число $x$-точек справа от каждого $y_{k}$ на единицу больше, чем число $y$-точек справа от $y_{k}$. Отсюда следует, что все коэффициенты $\nu_{k}$ положительны.

Предположим теперь, что коэффициенты $\nu_{k}$ положительны. Тогда каждые две смежные $y$-точки разделяются некоторой $x$-точкой (иначе соответствующие коэффициенты $\nu_{k}$ имели бы противоположные знаки). Поскольку $\nu_{d-1}>0$ и $\nu_{1}>0$, существуют хотя бы одна $x$-точка, превосходящая $y_{d-1}$, и хоть одна $x$-точка, меньшая $y_{1}$. Поэтому две последовательности перемежаются.

ОПРЕДЕЛЕНИЕ 2.4. Пусть $A=A(\omega)$ - площадь диаграммы $\omega$ (см. (1.4)), связанной с парой перемежающихся последовательностей (1.1). Обозначим через $\nu$ систему весов

$$
\frac{\nu_{k}}{A}=\frac{\left(x_{d}-y_{k}\right)\left(y_{k}-x_{1}\right)}{A} \prod_{i=1}^{k-1} \frac{y_{k}-x_{i+1}}{y_{k}-y_{i}} \prod_{j=k+1}^{d-1} \frac{y_{k}-x_{j}}{y_{k}-y_{j}}
$$

в точках $y_{k}, k=1, \ldots, d-1$. По лемме $2.3 \nu$ - вероятностное распределение. Мы называем $\nu$ копереходным распределением, а веса $\nu_{k} / A$ - копереходньли вероятностями. 


\section{§3. Копереходное распределение диаграммы Юнга}

Мы покажем в этом параграфе, что переходное и копереходное распределения, введенные в $\S 2$, обобщают соответствующие комбинаторные понятия. Напомним сначала определение процесса планшерелевского роста (см. подробности в [2] и [12]) и связанные с ним комбинаторные определения переходных и копереходных вероятностей.

Обозначим через $\mathbb{Y}_{n}$ множество диаграмм Юнга с $n$ клетками, так что $\mathbb{Y}=\bigcup \mathbb{Y}_{n}$ - решетка всех диаграмм Юнга, упорядоченных по включению. Мы пишем $\lambda \nearrow \Lambda$, если диаграмма $\Lambda$ накрывает $\lambda$ в $\mathbb{Y}$, т. е. если $\Lambda=\lambda \cup b_{0}$ есть объединение диаграммы $\lambda$ и дополнительной клетки $b_{0}$.

Пусть $\operatorname{dim} \lambda$ обозначает число стандартных таблиц Юнга формы $\lambda \in \mathbb{Y}$. Функцию размерности $\operatorname{dim} \lambda$ можно охарактеризовать начальным условием $\operatorname{dim} \varnothing=1$ и рекуррентным соотношением

$$
\operatorname{dim} \Lambda=\sum_{\lambda: \lambda \nearrow \Lambda} \operatorname{dim} \lambda
$$

(напоминающим алгоритм треугольника Паскаля), которое можно также переписать в форме

$$
\sum_{\lambda: \lambda \nearrow \Lambda} \frac{\operatorname{dim} \lambda}{\operatorname{dim} \Lambda}=1
$$

Отношения $q_{\Lambda}(\lambda)=\operatorname{dim} \lambda / \operatorname{dim} \Lambda$ называются копереходными вероятностями диаграммы $\Lambda$.

Согласно знаменитой формуле крюков [14],

$$
\operatorname{dim} \lambda=n ! \prod_{b \in \lambda} h^{-1}(b),
$$

где $h(b)=\left(\lambda_{i}-j\right)+\left(\lambda_{j}^{\prime}-i\right)+1$ называется длиной крюка клетки $b=(i, j)$ на пересечении $i$-й строки и $j$-го столбца.

Чтобы доказать формулу (3.2), достаточно проверить, что правая часть удовлетворяет рекуррентному соотношению, сходному с (3.1). Мы выводим этот факт из леммы 2.3. С этой целью мы прежде всего убедимся, что комбинаторное определение копереходных вероятностей эквивалентно аналитическому определению из $\S 2$.

Лемма 3.1. Обозначим через $x_{1}<y_{1}<\cdots<y_{d-1}<x_{d}$ перемежаюшиеся последовательности, связанные с диаграммой Юнга $\Lambda$ с А клетками (см. 1 ), и пусть $\lambda$ - диаграмма Юнга, полученная из А удалением клетки с содержанием $y_{k}$. Пусть $f_{\lambda}$ обозначает правую часть формуль (3.2). Тогда

$$
\frac{f_{\lambda}}{f_{\Lambda}}=\frac{\left(x_{d}-y_{k}\right)\left(y_{k}-x_{1}\right)}{A} \prod_{i=1}^{k-1} \frac{y_{k}-x_{i+1}}{y_{k}-y_{i}} \prod_{j=k+1}^{d-1} \frac{y_{k}-x_{j}}{y_{k}-y_{j}} .
$$

ДоКАЗАТЕЛЬСТво. По определению отношение в левой части можно записать в виде

$$
\frac{f_{\lambda}}{f_{\Lambda}}=\frac{1}{|\lambda|} \prod_{b} \frac{h(b)+1}{h(b)}
$$


где произведение берется по клеткам в столбце диаграммы $\lambda$ над новой клеткой $b_{0}=\Lambda \backslash \lambda$ и в ее строке слева от $b_{0}$. Длины крюков $h(b)$ берутся по отношению к меньшей диаграмме $\lambda$. Рассмотрим пересечение $I$ столбца, содержащего клетку $b_{0}$, с блоком равных строк диаграммы Юнга $\lambda$ (см. рис. 2а). Этот блок соответствует вертикальному отрезку на границе диаграммы, и мы обозначаем через $y_{j}, x_{j+1}$ содержания его концов. Если $j>k$, то произведение вдоль $I$ упрощается до

$$
\prod_{b \in I} \frac{h(b)+1}{h(b)}=\frac{y_{j}-y_{k}+1}{y_{j}-y_{k}} \frac{y_{j}-y_{k}+2}{y_{j}-y_{k}+1} \cdots \frac{x_{j+1}-y_{k}}{x_{j+1}-y_{k}-1}=\frac{x_{j+1}-y_{k}}{y_{j}-y_{k}} .
$$

Если же $j=k$, то минимальный крюк в $I$ равен единице и произведение сводится к $\prod_{b \in I}(h(b)+1) / h(b)=x_{k+1}-y_{k}$.

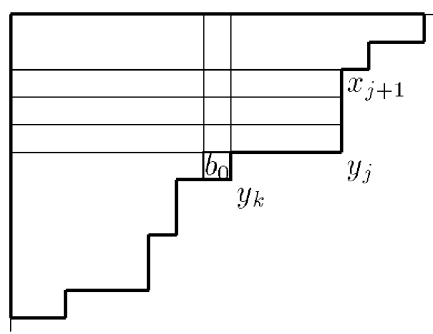

a)

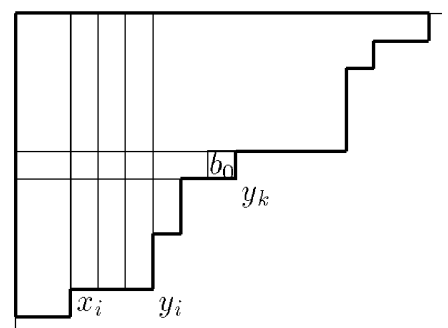

b)

\section{Рис. 2}

Аналогично мы рассматриваем случай, когда $I$ есть пересечение строки, содержащей новую клетку $b_{0}$, с блоком равных столбцов диаграммы $\lambda$ (см. рис. 2b). Такой блок соответствует горизонтальному отрезку на границе диаграммы $\lambda$ и мы обозначаем через $x_{i}, y_{i}$ содержания его концов. Произведение вдоль $I$ сводится в этом случае к

$$
\prod_{b \in I} \frac{h(b)+1}{h(b)}=\frac{y_{k}-y_{i}+1}{y_{k}-y_{i}} \frac{y_{k}-y_{i}+2}{y_{k}-y_{i}+1} \cdots \frac{y_{k}-x_{i}}{y_{k}-x_{i}-1}=\frac{y_{k}-x_{i}}{y_{k}-y_{i}},
$$

если $i<k$, и к $\prod_{I}(h+1) / h=y_{k}-x_{k}$, если $i=k$.

Формула крюков (3.2) непосредственно следует из лемм 2.3 и 3.1. Действительно, из них вытекает, что $\sum f_{\lambda} / f_{\Lambda}=1$, а потому числа $f_{\lambda}$ удовлетворяют тому же рекуррентному соотношению (3.1), что и $\operatorname{dim} \lambda$, и тождество $\operatorname{dim} \lambda=f_{\lambda}$ справедливо.

\section{§4. Планшерелевские переходные распределения}

Обсудим теперь хорошо известную формулу (см. [15, Eq. (B)])

$$
\sum_{\Lambda: \lambda \nearrow \Lambda} \operatorname{dim} \Lambda=(|\lambda|+1) \operatorname{dim} \lambda
$$

имеющую целый ряд различных доказательств. Например, можно подсчитать двумя различными способами размерность представления Ind $\pi_{\lambda}$ симметрической группы $\mathfrak{S}_{n+1}$, индуцированного неприводимым представлением $\pi_{\lambda}$ (помеченным диаграммой Юнга $\lambda$ ) подгруппы $\mathfrak{S}_{n}$. Доказательство при помощи 
явного построения биекции можно получить, опираясь на основные свойства алгоритма вставки Робинсона-Шенстеда (см. [16]). Здесь мы выведем (4.1) из того факта, что веса (2.2), ассоциированные с парой перемежающихся последовательностей, всегда образуют вероятностное распределение.

Формулу (4.1) можно переписать в форме

$$
\sum_{\Lambda: \lambda \nearrow \Lambda} \frac{\operatorname{dim} \Lambda}{(n+1) \operatorname{dim} \lambda}=1,
$$

так что числа $\mu_{\lambda}(\Lambda)=\operatorname{dim} \Lambda /(n+1) \operatorname{dim} \lambda($ где $\lambda \nearrow \Lambda$ и $n=|\lambda|)$ можно взять в качестве весов вероятностного распределения $\mu_{\lambda}$, связанного с диаграммой Юнга $\lambda \in \mathbb{Y}_{n}$.

Рассмотрим марковскую цепь на решетке Юнга, называемую процессом планшерелевского роста. По определению он начинается в пустой диаграмме $\varnothing$ и имеет переходные вероятности $p(\lambda, \Lambda)=\mu_{\lambda}(\Lambda)$. Можно легко проверить, что вероятность пересечь $n$-й этаж $\mathbb{Y}_{n}$ решетки Юнга в определенной диаграмме Юнга $\lambda$ равна $M_{n}(\lambda)=\operatorname{dim}^{2} \lambda / n$ !, а это есть вес указанной диаграммы по отношению к мере Планшереля группы $\mathfrak{S}_{n}$. В многих отношениях эту цепь можно рассматривать как меру Планшереля бесконечной симметрической группы $\mathfrak{S}_{\infty}$. Следуя [7], мы называем $\mu_{\lambda}$ планшерелевским переходным распределением диаграммы $\lambda$.

Процесс планшерелевского роста является чентральной марковской цепью. Это означает, что вероятность достичь диаграммы Юнга $\lambda$ вдоль определенного пути (т.е. таблицы Юнга) зависит только от конечной диаграммы $\lambda$, но не от выбора этого пути. В нашем случае эта вероятность равна $\operatorname{dim} \lambda / n$ !.

Напомним, что копереходная вероятность $q(\lambda, \Lambda)$ некоторой марковской цепи на решетке Юнга определяется как условная вероятность пройти через вершину $\lambda$ при условии, что следующей вершиной является $\Lambda$. Для каждой центральной марковской цепи эта копереходная вероятность равна $q(\lambda, \Lambda)=$ $\operatorname{dim} \lambda / \operatorname{dim} \Lambda$. В действительности эта последняя формула характеризует центральные марковские цепи.

Лемма 4.1. Обозначим через $x_{1}<y_{1}<x_{2}<\cdots<y_{d-1}<x_{d}$ перемежающиеся последовательности, ассочиированные с диаграммой Юнга $\lambda \in \mathbb{Y}_{n}$. Допустим, что диаграмма $\Lambda$, где $\lambda \nearrow \Lambda$, получаетсл из $\lambda$ добавлением клетки $b_{0}$ с содержанием $x_{k}$. Тогда

$$
\frac{\operatorname{dim} \Lambda}{(n+1) \operatorname{dim} \lambda}=\prod_{i=1}^{k-1} \frac{x_{k}-y_{i}}{x_{k}-x_{i}} \prod_{j=k+1}^{d} \frac{x_{k}-y_{j-1}}{x_{k}-x_{j}},
$$

т.е. комбинаторное и аналитическое определения переходных вероятностей эквивалентнь.

ДокАЗАТЕЛЬСтво. По формуле крюков (3.2) левую часть можно записать в виде

$$
\frac{\operatorname{dim} \Lambda}{(n+1) \operatorname{dim} \lambda}=\prod_{b} \frac{h(b)}{h(b)+1},
$$

где произведение берется по клеткам в строке и в столбце диаграммы Юнга $\lambda$, содержащих новую клетку $b_{0}$. Все длины крюков $h(b)$ берутся по отношению к меньшей диаграмме $\lambda$. 
Оставшаяся часть рассуждения совершенно такая же, как в доказательстве леммы 3.1. Рассмотрим, например, пересечение $I$ столбца, проходящего через клетку $b_{0}$, с блоком равных строк диаграммы $\lambda$. Пусть $y_{j-1}, x_{j}$ - содержания концов вертикального отрезка на пересечении этих строк с графиком диаграммы $\lambda$. Тогда

$$
\prod_{b \in I} \frac{h(b)}{h(b)+1}=\frac{y_{j-1}-x_{k}}{y_{j-1}-x_{k}+1} \frac{y_{j-1}-x_{k}+1}{y_{j-1}-x_{k}+2} \cdots \frac{x_{j}-x_{k}-1}{x_{j}-x_{k}}=\frac{x_{k}-y_{j-1}}{x_{k}-x_{j}} .
$$

Аналогичным образом мы поступаем с произведением вдоль части $I$ строки, содержащей $b_{0}$, соответствующей горизонтальному интервалу с концами $x_{i}, y_{i}$ на границе диаграммы $\lambda$. Это произведение равно

$$
\prod_{b \in I} \frac{h(b)}{h(b)+1}=\frac{x_{k}-y_{i}}{x_{k}-y_{i}+1} \frac{x_{k}-y_{i}+1}{x_{k}-y_{i}+2} \cdots \frac{x_{k}-x_{i}-1}{x_{k}-x_{i}}=\frac{x_{k}-y_{i}}{x_{k}-x_{i}}
$$

откуда и следует лемма.

\section{§5. Переходные распределения $z$-мер}

В этом параграфе мы находим моменты переходного распределения (2.2) пары перемежающихся последовательностей. Затем мы используем эти моменты, чтобы определить семейство центральных марковских цепей на решетке Юнга, введенное в [6].

Пусть $\mu$ - переходное распределение (2.2) пары перемежающихся последовательностей $x_{1}<y_{1}<x_{2}<\cdots<x_{d}$, и пусть

$$
h_{m}=\sum_{k=1}^{d} x_{k}^{m} \mu_{k}
$$

есть $m$-й момент для $\mu$. Мы рассматриваем производящую функцию

$$
R(u)=\sum_{m=0}^{\infty} \frac{h_{m}}{u^{m+1}}
$$

ЛЕмма 5.1. Производяиая Функиия моментов (5.2) равна

$$
\sum_{m=0}^{\infty} \frac{h_{m}}{u^{m+1}}=\frac{\left(u-y_{1}\right) \cdots\left(u-y_{d-1}\right)}{\left(u-x_{1}\right) \cdots\left(u-x_{d-1}\right)\left(u-x_{d}\right)}
$$

ДокАЗАТЕльСтво. Поскольку $1 /\left(u-x_{k}\right)=\sum_{m \geqslant 0} x_{k}^{m} / u^{m+1}$, мы получаем, что

$$
R(u)=\sum_{k=1}^{d} \frac{\mu_{k}}{u-x_{k}},
$$

и утверждение следует из тождества (2.1).

СлЕДСТВИЕ 5.2. Пусть с и А обозначают иентр и площадь диаграммь, ассоииированной с перемежающимися последовательностями $(X, Y)$. Тогда среднее значение их переходного распределения есть $h_{1}=c$, a дисперсия равна $h_{2}-h_{1}^{2}=$ A. Следовательно, первье два момента (переходного распределения) диаграммы Юнга зависят только от числа ее клеток. 
ДоКАЗАТЕЛЬСТвО. Используя обозначение $\S 2$ для элементарных симметрических функций, мы выводим из формулы (5.3), что $h_{1}=e_{1}(X)-e_{1}(Y)=c$ и $h_{2}=e_{1}^{2}(X)-e_{2}(X)-e_{1}(X) e_{1}(Y)+e_{2}(Y)$. Следовательно, $h_{2}-h_{1}^{2}=-e_{2}(X)+$ $e_{1}(X) e_{1}(Y)+e_{2}(Y)-e_{1}^{2}(Y)=A$.

СледСТВИЕ 5.3. Для заданных комплексных параметров и и $v$ рассмотрим числа

$$
p_{u, v}(\lambda, \Lambda)=\frac{(c(b)+u)(c(b)+v)}{n+u v} \frac{\operatorname{dim} \Lambda}{(n+1) \operatorname{dim} \lambda},
$$

где $\lambda \in \mathbb{Y}_{n}, \lambda \nearrow \Lambda u b=\Lambda \backslash \lambda$. Тогда

$$
\sum_{\Lambda: \lambda \nearrow \Lambda} p_{u, v}(\lambda, \Lambda)=1
$$

Если $v=\bar{u}$, то числа (5.4) положительны и их можно взять в качестве переходных вероятностей марковской цепи на решетке Юнга. Все такие цепи центральны. В пределе при $u \rightarrow \infty$ мы получаем процесс планшерелевского роста, так что это семейство можно рассматривать как деформацию меры Планшереля бесконечной симметрической группы $\mathfrak{S}_{\infty}$.

\section{§6. Копереходные вероятности, определяемые симметрическими функциями Джека}

Следуя [9, Ch. VI.10], мы обозначаем через $P_{\lambda}(x)=P_{\lambda}\left(x_{1}, x_{2}, \ldots ; \alpha\right), \lambda \in \mathbb{Y}$, семейство симметрических функций Джека с параметром $\alpha$. При фиксированном значении $\alpha$ функции $P_{\lambda}$ образуют линейный базис в алгебре симметрических функций.

Обозначим через $p_{1}(x)=x_{1}+x_{2}+\cdots$ сумму всех переменных и рассмотрим разложение произведения $p_{1} P_{\lambda}$ по базису $P_{\Lambda}$. Известно [9, VI.6.24(iv), V.10.10], что

$$
p_{1}(x) P_{\lambda}(x ; \alpha)=\sum_{\Lambda: \lambda \nearrow \Lambda} \varkappa_{\alpha}(\lambda, \Lambda) P_{\Lambda}(x ; \alpha),
$$

где коэффициенты (кратности) $\varkappa_{\alpha}(\lambda, \Lambda)$ задаются явной формулой:

$$
\varkappa_{\alpha}(\lambda, \Lambda)=\prod_{b \in \mathrm{ver}} \frac{(a(b) \alpha+l(b)+2)(a(b) \alpha+l(b)+1)}{((a(b)+1) \alpha+l(b)+1)(a(b) \alpha+l(b)+1)} .
$$

Здесь $b$ пробегает все клетки $j$-го столбца ver диаграммы $\lambda$ при условии, что новая клетка $b_{0}=\Lambda \backslash \lambda$ принадлежит $j$-му столбцу диаграммы $\Lambda$. Число $a(i, j)=$ $\lambda_{i}-j$ называется длиной руки, а $l(i, j)=\lambda_{j}^{\prime}-i-$ длиной ноги клетки $b=(i, j)$ в диаграмме Юнга $\lambda$.

Мы определяем обобщение $\operatorname{dim}_{\alpha} \lambda$ функции размерности $\operatorname{dim} \lambda$ посредством рекуррентной формулы

$$
\operatorname{dim}_{\alpha} \Lambda=\sum_{\lambda: \lambda \nearrow \Lambda} \varkappa_{\alpha}(\lambda, \Lambda) \operatorname{dim}_{\alpha} \lambda
$$

с начальным условием $\operatorname{dim}_{\alpha}(\varnothing)=1$. Если $\alpha=1$, то кратности тривиальны, $\varkappa_{1}(\lambda, \Lambda) \equiv 1$, и $\operatorname{dim}_{1} \lambda=\operatorname{dim} \lambda$ есть обычная функция размерности. Полиномы Джека в этом случае совпадают с обычными функциями Шура, $P_{\lambda}(x ; 1) \equiv$ $s_{\lambda}(x)$. 
Формула

$$
\operatorname{dim}_{\alpha} \lambda=n ! \alpha^{n} \prod_{b \in \lambda} \frac{1}{(a(b)+1) \alpha+l(b)}
$$

для функции размерности $\operatorname{dim}_{\alpha} \lambda,|\lambda|=n$, обобщающая формулу крюков, была найдена Стенли [10]. Мы выведем (6.4) как частный случай леммы 2.3.

Для этой цели мы обозначаем через $D_{\alpha}$ преобразование растяжения $D_{\alpha}(u, v)$ $=(u, v \alpha),(u, v) \in \mathbb{R}^{2}$, и рассматриваем образ $\Lambda^{\alpha}=D_{\alpha}(\Lambda)$ диаграммы Юнга $\Lambda$ при этом растяжении. Заметим, что $\alpha$-содержание $c_{\alpha}(u, v)=v \alpha-u$ точки $(u, v)$ совпадает с обычным содержанием $c(u, v \alpha)$ соответствующей точки $D_{\alpha}(u, v)$. Мы обозначаем через

$$
x_{1}(\alpha)<y_{1}(\alpha)<x_{2}(\alpha)<\cdots<x_{d-1}(\alpha)<y_{d-1}(\alpha)<x_{d}(\alpha)
$$

пару перемежающихся последовательностей, соответствующую растянутой диаграмме $\Lambda^{\alpha}$. Другими словами, (6.5) - это последовательность $\alpha$-содержаний угловых точек первоначальной диаграммы Юнга $\Lambda$. Центр пары (6.5) тривиален, $c\left(\Lambda^{\alpha}\right)=0$, а ее площадь равна $A\left(\Lambda^{\alpha}\right)=\alpha|\Lambda|$.

ОПРЕДЕЛЕНИЕ 6.1. Пусть $\lambda, \Lambda$ - такие диаграммы Юнга, что $\lambda \nearrow \Lambda$, и предположим, что $\alpha>0$. Согласно (6.3), числа $\varkappa_{\alpha}(\lambda, \Lambda) \operatorname{dim}_{\alpha} \lambda / \operatorname{dim}_{\alpha} \Lambda$ можно рассматривать как вероятности. Мы называем их $\alpha$-копереходными вероятностями диаграммы $\Lambda$.

Теорема 6.2. Предположим, ито диаграмма Юнга $\lambda$ получена из $\Lambda \in \mathbb{Y}_{n}$ удалением клетки $b_{0}$ c $\alpha$-содержанием $y_{k}(\alpha)$ (более точно, это $\alpha$-содержание юго-восточного угла клетки). Тогда

$$
\begin{aligned}
\frac{\varkappa_{\alpha}(\lambda, \Lambda) \operatorname{dim}_{\alpha} \lambda}{\operatorname{dim}_{\alpha} \Lambda}= & \frac{\left(x_{d}(\alpha)-y_{k}(\alpha)\right)\left(y_{k}(\alpha)-x_{1}(\alpha)\right)}{A\left(\Lambda^{\alpha}\right)} \\
& \times \prod_{i=1}^{k-1} \frac{y_{k}(\alpha)-x_{i+1}(\alpha)}{y_{k}(\alpha)-y_{i}(\alpha)} \prod_{j=k+1}^{d-1} \frac{y_{k}(\alpha)-x_{j}(\alpha)}{y_{k}(\alpha)-y_{j}(\alpha)},
\end{aligned}
$$

т.е. $\alpha$-копереходные вероятности диаграммы Юнга $\Lambda$ совпадают с соответствуюшими копереходными вероятностями ассочиированной пары перемежающихся последовательностей (6.5).

ДоказАТЕльство. Оно очень похоже на доказательство леммы 3.1. Согласно $\alpha$-формуле крюков (6.4),

$$
\frac{\operatorname{dim}_{\alpha} \lambda}{\operatorname{dim}_{\alpha} \Lambda}=\frac{1}{n} \prod_{b \in \text { ver }} \frac{(a(b)+1) \alpha+l(b)+1}{(a(b)+1) \alpha+l(b)} \prod_{b \in \text { hor }} \frac{(a(b)+2) \alpha+l(b)}{(a(b)+1) \alpha+l(b)},
$$

где ver обозначает столбец диаграммы $\lambda$ над новой клеткой $b_{0}=\Lambda \backslash \lambda$, hor это строка диаграммы $\lambda$ слева от $b_{0}$, а длины рук и ног берутся по отношению к меньшей диаграмме $\lambda$. Мы также учитываем $\alpha$-крюк $h_{\alpha}\left(b_{0}\right)=(a(b)+1) \alpha+l(b)=$ $\alpha$ клетки $b_{0}$.

Комбинируя это вычисление с формулой кратностей (6.2), мы получаем, что

$$
\frac{\varkappa_{\alpha}(\lambda, \Lambda) \operatorname{dim}_{\alpha} \lambda}{\operatorname{dim}_{\alpha} \Lambda}=\frac{1}{n} \prod_{b \in \text { ver }} \frac{a(b) \alpha+l(b)+2}{a(b) \alpha+l(b)+1} \prod_{b \in \text { hor }} \frac{(a(b)+2) \alpha+l(b)}{(a(b)+1) \alpha+l(b)} .
$$


Как и при доказательстве леммы 3.1, рассмотрим пересечение $I$ столбца ver с блоком равных строк диаграммы $\lambda$. Заметим, что длины рук клеток из $I$ одинаковы, а длины ног возрастают на 1. Предполагая, что строки из $I$ соответствуют интервалу $\left(y_{j}(\alpha), x_{j+1}(\alpha)\right)$, получаем, что

$$
\prod_{I} \frac{a(b) \alpha+l(b)+2}{a(b) \alpha+l(b)+1}= \begin{cases}\frac{x_{j+1}(\alpha)-y_{k}(\alpha)}{y_{j}(\alpha)-y_{k}(\alpha)}, & \text { если } j>k, \\ x_{k+1}(\alpha)-y_{k}(\alpha), & \text { если } j=k .\end{cases}
$$

Для части $I$ строки hor, соответствующей интервалу $\left(x_{i}(\alpha), y_{i}(\alpha)\right)$, мы получаем

$$
\prod_{I} \frac{(a(b)+2) \alpha+l(b)}{(a(b)+1) \alpha+l(b)}= \begin{cases}\frac{y_{k}(\alpha)-x_{i}(\alpha)}{y_{k}(\alpha)-y_{i}(\alpha)}, & \text { если } i<k, \\ \frac{y_{k}(\alpha)-x_{k}(\alpha)}{\alpha}, & \text { если } i=k .\end{cases}
$$

Поскольку $A\left(\Lambda^{\alpha}\right)=n \alpha,(6.6)$ следует из (6.7) и теорема доказана.

В ходе доказательства теоремы мы воспользовались $\alpha$-формулой крюков (6.4) как определением $\alpha$-функции размерности. Мы совершенно не применяли рекуррентную формулу (6.3). И наоборот, формула крюков (6.4) в действительности является прямым следствием теоремы 6.2 и леммы 2.3. В самом деле, из леммы 2.3 следует, что функция, определенная посредством (6.4), удовлетворяет рекуррентному соотношению (6.3), а потому оба определения эквивалентны.

СлЕдСТВИЕ 6.3 (Стенли [10]). Для функиии $\operatorname{dim}_{\alpha} \lambda$, определенной посредством (6.3), справедлива формула крюков (6.4).

\section{§7. Процесс планшерелевского роста для анизотропных диаграмм}

В предыдущем параграфе мы рассматривали систему кратностей $\varkappa_{\alpha}(\lambda, \Lambda)$ для дуг графа Юнга, зависящую от параметра $\alpha>0$. Эти кратности определяют в силу формулы (6.3) систему копереходных вероятностей. По теореме 6.2 те же вероятности

$$
q_{\alpha}(\lambda, \Lambda)=\frac{\varkappa_{\alpha}(\lambda, \Lambda) \operatorname{dim}_{\alpha} \lambda}{\operatorname{dim}_{\alpha} \Lambda}, \quad \lambda: \lambda \nearrow \Lambda,
$$

возникают также из общего определения 2.4, если заменить каждую диаграмму Юнга $\Lambda$ на ее растянутую версию $\Lambda^{\alpha}$. Теперь мы хотим описать замечательную марковскую цепь на решетке Юнга, центральную по отношению к копереходным вероятностям (7.1). Эта цепь окажется обобщением процесса планшерелевского роста для растянутых (или анизотропныл) диаграмм Юнга.

ЛЕмма 7.1. Рассмотрим функиию $\varphi: \mathbb{Y} \rightarrow \mathbb{R}$, определенную посредством $\oint о р м у л ь \imath$

Tozдa

$$
\varphi(\lambda)=\prod_{b \in \lambda} \frac{1}{a(b) \alpha+l(b)+1} .
$$

$$
\frac{\varkappa_{\alpha}(\lambda, \Lambda) \varphi(\Lambda)}{\varphi(\lambda)}=\prod_{i=1}^{k-1} \frac{x_{k}(\alpha)-y_{i}(\alpha)}{x_{k}(\alpha)-x_{i}(\alpha)} \prod_{j=k+1}^{d} \frac{x_{k}(\alpha)-y_{j-1}(\alpha)}{x_{k}(\alpha)-x_{j}(\alpha)}
$$

для яюбой диаграммью Юнга $\lambda$. 
ДокАЗАТЕЛьство. Из формулы кратностей (6.2) следует, что

$$
\frac{\varkappa_{\alpha}(\lambda, \Lambda) \varphi(\Lambda)}{\varphi(\lambda)}=\prod_{b \in \mathrm{ver}} \frac{(a(b)+1) \alpha+l(b)}{(a(b)+1) \alpha+l(b)+1} \prod_{b \in \mathrm{hor}} \frac{a(b) \alpha+l(b)+1}{(a(b)+1) \alpha+l(b)+1}
$$

где hor и ver были определены в доказательстве теоремы 6.2.

Обозначим через

$$
x_{1}(\alpha)<y_{1}(\alpha)<x_{2}(\alpha)<\cdots<x_{d-1}(\alpha)<y_{d-1}(\alpha)<x_{d}(\alpha)
$$

перемежающиеся последовательности, соответствующие растянутой форме $\lambda^{\alpha}$, и предположим, что $\alpha$-содержание (северо-западного угла) новой клетки $b_{0}=$ $\Lambda \backslash \lambda$ равно $x_{k}(\alpha)$.

Продолжая, как в доказательстве леммы 4.1, мы расшепляем столбец ver на блоки клеток с одинаковой длиной руки. Тогда $\alpha$-крюки $(a(b)+1) \alpha+l(b)$ в таком блоке $I$ меняются от $y_{j-1}(\alpha)-x_{k}(\alpha)$ до $x_{j}(\alpha)-x_{k}(\alpha)-1$ при некотором $j>k$. Следовательно,

$$
\begin{aligned}
& \prod_{b \in I} \frac{(a(b)+1) \alpha+l(b)}{(a(b)+1) \alpha+l(b)+1}=\frac{x_{k}(\alpha)-y_{j-1}(\alpha)}{x_{k}(\alpha)-x_{j}(\alpha)} \\
& \prod_{b \in \text { ver }} \frac{(a(b)+1) \alpha+l(b)}{(a(b)+1) \alpha+l(b)+1}=\prod_{j=k+1}^{d} \frac{x_{k}(\alpha)-y_{j-1}(\alpha)}{x_{k}(\alpha)-x_{j}(\alpha)} .
\end{aligned}
$$

Точно так же

$$
\prod_{b \in \text { hor }} \frac{a(b) \alpha+l(b)+1}{(a(b)+1) \alpha+l(b)+1}=\prod_{i=1}^{k-1} \frac{x_{k}(\alpha)-y_{i}(\alpha)}{x_{k}(\alpha)-x_{i}(\alpha)}
$$

откуда и следует формула (7.3).

Tеорема 7.2. Числа $p_{\alpha}(\lambda, \Lambda)=\varkappa_{\alpha}(\lambda, \Lambda) \varphi(\Lambda) / \varphi(\lambda), \lambda \nearrow \Lambda$, образуют систему переходных вероятностей для некоторой марковской чепи на графе Юнаа, иентральной по отношению к кратностям дуг (6.2).

ДоказАТЕльство. Согласно формуле (7.3) и лемме 2.1 , числа $p_{\alpha}(\lambda, \Lambda)$ образуют вероятностное распределение для любой фиксированной диаграммы $\lambda$. Вероятность достичь заданную диаграмму Юнга $\Lambda \in \mathbb{Y}_{n}$ вдоль определенной таблицы Юнга $t=\left(\varnothing=\lambda_{0} \subset \lambda_{1} \subset \cdots \subset \lambda_{n}=\Lambda\right)$ равна, как легко видеть,

$$
P_{\alpha}(t)=\varphi(\Lambda) \prod_{k=1}^{n} \varkappa_{\alpha}\left(\lambda_{k-1}, \lambda_{k}\right)
$$

Следовательно, копереходные вероятности $q(\lambda, \Lambda)$ равны $\varkappa_{\alpha}(\lambda, \Lambda) \operatorname{dim}_{\alpha} \lambda / \operatorname{dim}_{\alpha} \Lambda$ для любой диаграммы $\Lambda$ и цепь центральна.

Аналогично следствию 5.3 мы выводим из (7.3) и следствия 5.2, что сумма чисел

$$
p_{u, v}(\lambda, \Lambda)=\frac{\left(c_{\alpha}(b)+u\right)\left(c_{\alpha}(b)+v\right)}{n \alpha+u v} \frac{\varkappa_{\alpha}(\lambda, \Lambda) \varphi(\Lambda)}{\varphi(\lambda)}
$$

равна единице. Если все эти числа положительны (например, если $v=\bar{u}$ ), то можно определить марковскую цепь на решетке Юнга с переходными вероятностями (7.4). 
СлЕдСТВИЕ 7.3. Марковская чепь с переходньми вероятностями (7.4) иентральна по отношению $\kappa$ копереходньм вероятностям (7.1).

ДокАЗАТЕльство. Для каждой таблицы Юнга $\varnothing=\lambda_{0} \subset \lambda_{1} \subset \cdots \subset \lambda_{n}=\Lambda$ произведение

$$
\prod_{k=1}^{n} \frac{p_{u, v}\left(\lambda_{k-1}, \lambda_{k}\right)}{\varkappa_{\alpha}\left(\lambda_{k-1}, \lambda_{k}\right)}=\frac{\varphi(\Lambda)}{u v(u v+\alpha) \cdots(u v+(n-1) \alpha)} \prod_{b \in \lambda}\left(c_{\alpha}(b)+u\right)\left(c_{\alpha}(b)+v\right)
$$

зависит только от последней диаграммы Юнга $\Lambda$ (т.е. от формы таблицы). Это и означает, что наша марковская цепь центральна.

\section{ЛитерАТУРА}

1. Kerov $S$. $V$. A $q$-analog of the hook walk algorithm for random Young tableaux. J. Algebraic Combin., 2, 383-396 (1993).

2. Kerov $S$. $V$. The boundary of Young lattice and random Young tableaux. DIMACS Ser. Discrete Math. Theoret. Comput. Sci., 24, 133-158 (1996).

3. Керов C. В. Асимптотика разделения корней ортогональных многочленов. Алгебра и анализ, 5, вып. 5, 68-86 (1993).

4. Керов $C$. $B$. Переходные вероятности континуальных диаграмм Юнга и проблема моментов Маркова. Функц. анализ и его прил., 27, вып. 2, 32-49 (1993).

5. Kerov S. V. Interlacing measures. Amer. Math. Soc. Transl. Ser. 2 (Adv. in Math. Sci., vol. 35 (G. I. Olshanski, ed.)), vol. 181, Providence, RI, 1998, pp. 35-83.

6. Kerov S. V., Olshanski G. I., Vershik A. M. Harmonic analysis on the infinite symmetric group. C. R. Acad. Sci. Paris Sér. I Math., 316, 773-778 (1993).

7. Kerov S. V., Vershik A. M. The Grothendieck group of the infinite symmetric group and symmetric functions with the elements of the $K_{0}$-functor theory of AF-algebras. In: Representation of Lie groups and related topics. Adv. Stud. Contemp. Math., vol. 7 (A. M. Vershik and D. P. Zhelobenko, eds.), Gordon and Breach, 1990, pp. 36-114.

8. Кириллов A. H. Тождество Лагранжа и формула крюков. Зап. семин. ЛОМИ, 172, 78-87 (1989).

9. Macdonald I. G. Symmetric functions and Hall polynomials. 2nd edition, Oxford University Press, 1995.

10. Stanley R. P. Some combinatorial properties of Jack symmetric functions. Adv. Math., 77, 76-115 (1989).

11. Вершик A. М. Формула крюков и связанные с ней тождества. Зап. семин. ЛОМИ, 172, 3-20 (1989).

12. Вершик A. М., Керов C. В. Асимптотика максимальной и типичной размерностей неприводимых представлений симметрической группы. Функц. анализ и его прил., 19, вып. 1, 25-36 (1985).

13. Kerov S. V., Okounkov A. and Olshanski G. I. The boundary of Young graph with Jack edge multiplicities. Internat. Math. Res. Notices, 4, 173-199 (1998) (q-alg/9703037).

14. Frame J. S., Robinson G. de B., Thrall R. M. The hook graphs of the symmetric group. Canad. J. Math., 6, 316-324 (1954).

15. Rutherford D. E. On the relations between the numbers of standard tableaux. Proc. Edinburgh Math. Soc., 7, 51-54 (1942-46).

16. Shensted $C$. Longest increasing and decreasing subsequences. Canad. J. Math., 13, 179-191 (1961).

Математический институт им. Стеклова (ПОМИ) email: kerov@pdmi.ras.ru 